\title{
Zero-forcing based multi-user MIMO coordinated with user-wise joint transmit- receive diversity
}

\author{
Fumiyuki Adachi ${ }^{1, \text { a) }}$ and Ryo Takahashi ${ }^{1}$ \\ ${ }^{1}$ Research Organization of Electrical Communication (ROEC), Tohoku University \\ 2-1-1 Katahira, Aoba-ku, Sendai, Miyagi, 980-8577 Japan \\ a)adachi@ecei.tohoku.ac.jp
}

Abstract: Multi-user MIMO multiplexing and spatial diversity are indispensable techniques to improve the link capacity in a fading environment. In this paper, user-wise joint transmit-receive diversity (JTRD) is proposed for zero-forcing (ZF)-based multi-user MIMO. Two types of user-wise JTRD are considered and the design of ZF precoder/postcoder, which takes into account the role of JTRD, is presented. The achievable received signal-to-noise ratio (SNR) is analyzed and a simple but useful expression for the received SNR is derived for a 2-user case. The link capacity achievable by ZF-based multiuser MIMO coordinated with user-wise JTRD in an uncorrelated Rayleigh fading environment is evaluated by Monte-Carlo numerical computation and is compared with ZF-based multi-user MIMO coordinated with user-wise maximal-ratio transmitting and combining diversity (MRTCD).

Keywords: multi-user MIMO multiplexing, joint transmit-receive diversity, zero-forcing

Classification: Wireless Communication Technologies

\section{References}

[1] L. Sanguinetti and H.V. Poor, "Fundamentals of multi-user MIMO communications," ed. V. Tarokh, New Directions in Wireless Communications Research, Chap. 6, Springer, Aug. 2009. DOI: 10.1007/978-1-4419-0673-1_6

[2] W.C. Jakes and D.C.Cox, eds., Microwave Mobile Communications, Wiley-IEEE Press, 1994.

[3] J.K. Cavers, "Single-user and multiuser adaptive maximal ratio transmission for Rayleigh channels," IEEE Trans. Veh. Technol., vol. 49, no. 6, pp. 2043-2050, Nov. 2000. DOI: 10.1109/25.901862

[4] Q.H. Spencer, C.B. Peel, A.L. Swindlehurst, and M. Haardt, "An introduction to the multi-user MIMO downlink," IEEE Commun. Mag., vol. 42, no. 10, pp. 6067, Oct. 2004. DOI: $10.1109 / \mathrm{mcom} .2004 .1341262$

[5] Q.H. Spencer, A.L. Swindlehurst, and M. Haardt, "Zero-forcing methods for downlink spatial multiplexing in multiuser MIMO channels," IEEE Trans. Signal Process., vol. 52, no. 2, pp. 461-471, Feb. 2004. DOI: 10.1109/tsp.2003.821107

[6] F. Adachi and R. Takahashi, "Analysis of 2-user ZF coordinated with user-wise MRTC diversity," IEICE Commun. Express, vol. 9, no. 9, pp. 433-439, Sept. 2020. DOI: $10.1587 /$ comex.2020xb10084 
[7] F. Adachi and A. Boonkajay, "Analysis of maximal-ratio transmit and combining spatial diversity," IEICE Commun. Express, vol. 8, no. 5, pp. 153-159, May 2019. DOI: 10.1587/comex.2019xbl0015

[8] A.M. Tulino and S. Verdu, Random Matrix Theory and Wireless Communications, Now Publishings, 2004. DOI: 10.1561/9781933019505

\section{Introduction}

Multi-user multi-input multi-output (MIMO) spatial multiplexing [1] has been recognized as an indispensable technique for efficiently utilizing the limited bandwidth. Meanwhile, spatial diversity [2,3] still remains an indispensable countermeasure against multipath fading. A well-known technique to remove the inter-user and inter-antenna interference produced by multi-user MIMO is the zero-forcing (ZF) based multi-user multiplexing employed at the base station (BS). In [4, 5], ZF-based multi-user MIMO downlink using joint transmitter-receiver beamforming was proposed. The BS must acquire the knowledge of multi-user MIMO channel to obtain the optimal transmitter and receiver processing matrices and must inform users of the receiver processing matrices.

Recently, we studied ZF-based multi-user MIMO coordinated with user-wise maximal-ratio transmitting and combining diversity (MRTCD) assuming timedivision duplex (TDD) downlink and uplink transmissions [6]. In this paper, we extend our previous study and propose user-wise joint transmit-receive diversity (JTRD) for ZF-based multi-user MIMO. JTRD was originally studied in [7] as single-user MIMO spatial diversity. Two types of user-wise JTRD are considered and the design of $\mathrm{ZF}$ precoder/postcoder, which takes into account the role of JTRD, is presented. The achievable received signal-to-noise ratio (SNR) is analyzed. A simple but useful expression, which helps to well understand ZF-based multi-user MIMO coordinated with user-wise JTRD, is derived for a 2-user case. The link capacity achievable by ZF-based multi-user MIMO coordinated with user-wise JTRD in an uncorrelated Rayleigh fading environment is evaluated by Monte-Carlo numerical computation and is compared with ZF-based multi-user MIMO coordinated with user-wise MRTCD.

In this paper, the superscripts $T, H$, and $*$ represent the transpose, Hermitian transpose, and complex conjugate operations, respectively. $E[$.$] and \|.\|_{2}$ represent the ensemble average operation and Frobenius norm, respectively.

\section{User-wise JTRD}

The transmission system model using user-wise JTRD is presented. Then, the procedure to determine the user-wise JTRD weight vectors is described. Finally, the $\mathrm{ZF}$ precoder/postcoder to remove the multi-user interference when user-wise JTRD is incorporated is derived.

\subsection{Transmission system model}

Downlink transmission system model for multi-user MIMO coordinated with userwise JTRD is illustrated in Fig. 1. The number of users is represented by $U$. BS has $M$ 


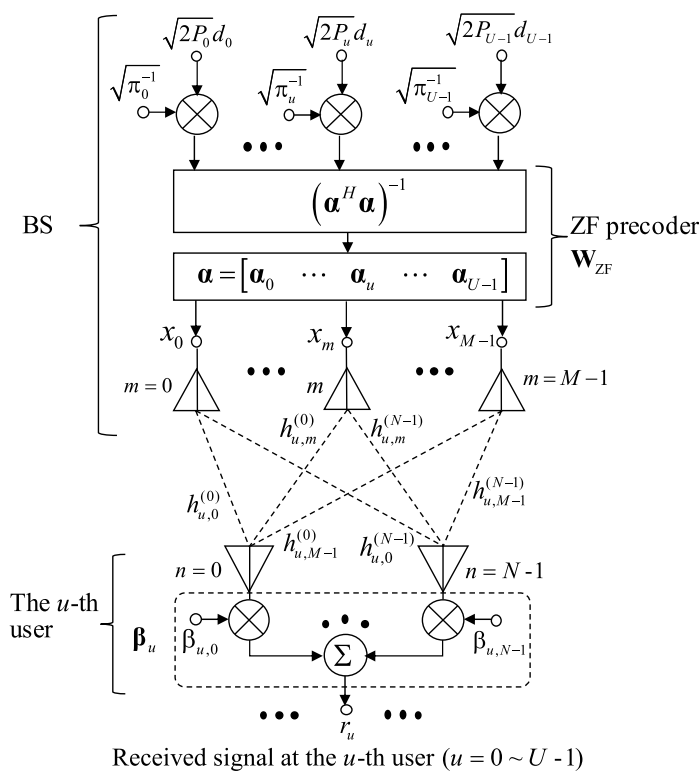

Fig. 1. Downlink transmission system model for multi-user MIMO coordinated with user-wise JTRD.

antennas and each user has $N$ antennas. The complex-valued fading gain between the $m(=0 \sim M-1)$-th BS antenna and the $u(=0 \sim U-1)$-th user's $n(=0 \sim N-1)$-th antenna is represented by $h_{u, m}^{(n)}$ with $E\left[\left|h_{u, m}^{(n)}\right|^{2}\right]=1$. The complete multi-user MIMO channel matrix is represented by $\mathbf{H}=\left[\begin{array}{lllll}\mathbf{H}_{0}^{T} & \cdots & \mathbf{H}_{u}^{T} & \cdots & \mathbf{H}_{U-1}^{T}\end{array}\right]^{T}$, where $\mathbf{H}_{u}=$ $\left[h_{u, m}^{(n)}\right]_{n=0 \sim N-1, m=0 \sim M-1}$ is the $u$-th user's single-user MIMO channel matrix. The user-wise JTRD weight vectors associated with the $u$-th user are represented as $\boldsymbol{\alpha}_{u}=$ $\left[\begin{array}{lllll}\alpha_{0, u} & \cdots & \alpha_{m, u} & \cdots & \alpha_{M-1, u}\end{array}\right]^{T}$ and $\boldsymbol{\beta}_{u}=\left[\begin{array}{lllll}\beta_{u, 0} & \cdots & \beta_{u, n} & \cdots & \beta_{u, N-1}\end{array}\right]$ with $\left\|\boldsymbol{\alpha}_{u}\right\|_{2}^{2}=\left\|\boldsymbol{\beta}_{u}\right\|_{2}^{2}=1 . \boldsymbol{\alpha}=\left[\begin{array}{lllll}\boldsymbol{\alpha}_{0} & \cdots & \boldsymbol{\alpha}_{u} & \cdots & \boldsymbol{\alpha}_{U-1}\end{array}\right]$ is a set of JTRD weight vectors to be used at BS and a concatenation of $\boldsymbol{\alpha}$ and $\left(\boldsymbol{\alpha}^{H} \boldsymbol{\alpha}\right)^{-1}$ constitutes ZF precoder $\mathbf{W}_{\text {ZF }}$ to remove the downlink inter-user interference. The user-wise downlink transmit power normalization factor $\pi_{u}$ is introduced to keep the $u$-th user's downlink transmit power intact after ZF precoding.

\subsection{Determining of $\alpha_{u}$ and $\beta_{u}$}

The user-wise JTRD is designed to maximize the composite channel gain $h_{u}=$ $\boldsymbol{\beta}_{u} \mathbf{H}_{u} \boldsymbol{\alpha}_{u}$. For the given $\boldsymbol{\beta}_{u}$ and $\mathbf{H}_{u}, \boldsymbol{\alpha}_{u}$ is given from Cauchy-Schwarz inequality [2] as

$$
\boldsymbol{\alpha}_{u}=\left(\boldsymbol{\beta}_{u} \mathbf{H}_{u}\right)^{H} / \sqrt{\left(\boldsymbol{\beta}_{u} \mathbf{H}_{u}\right)\left(\boldsymbol{\beta}_{u} \mathbf{H}_{u}\right)^{H}},
$$

from which we obtain $h_{u}=\boldsymbol{\beta}_{u}\left(\mathbf{H}_{u} \mathbf{H}_{u}^{H}\right) \boldsymbol{\beta}_{u}^{H} / \sqrt{\left(\boldsymbol{\beta}_{u} \mathbf{H}_{u}\right)\left(\boldsymbol{\beta}_{u} \mathbf{H}_{u}\right)^{H}}$. We want to find $\boldsymbol{\beta}_{u}$ which maximizes $h_{u} . \boldsymbol{\beta}_{u}$ is the solution of the following eigenvalue equation [8].

$$
\left(\mathbf{H}_{u} \mathbf{H}_{u}^{H}\right) \boldsymbol{\beta}_{u}^{H}=\omega_{u}^{+} \boldsymbol{\beta}_{u}^{H} \quad \text { s.t. }\left\|\boldsymbol{\beta}_{u}\right\|_{2}^{2}=1,
$$

where $\omega_{u}^{+}$is the maximum eigenvalue. Multiplying $\mathbf{H}_{u}^{H}$ to both side of Eq. (2) from the left and using Eq. (1), the following eigenvalue equation to determine $\boldsymbol{\alpha}_{u}$ is obtained.

$$
\left(\mathbf{H}_{u}^{H} \mathbf{H}_{u}\right) \boldsymbol{\alpha}_{u}=\omega_{u}^{+} \boldsymbol{\alpha}_{u} \quad \text { s.t. }\left\|\boldsymbol{\alpha}_{u}\right\|_{2}^{2}=1
$$


Furthermore, using Eqs. (1) and (2), the following useful equations are also obtained.

$$
\begin{aligned}
& \boldsymbol{\alpha}_{u}=\left(\boldsymbol{\beta}_{u} \mathbf{H}_{u}\right)^{H} / \sqrt{\omega_{u}^{+}}, \\
& \boldsymbol{\beta}_{u}=\left(\mathbf{H}_{u} \boldsymbol{\alpha}_{u}\right)^{H} / \sqrt{\omega_{u}^{+}}, \\
& \boldsymbol{\alpha}_{u}^{H} \mathbf{H}_{u}^{H} \mathbf{H}_{u} \boldsymbol{\alpha}_{u}=\boldsymbol{\beta}_{u} \mathbf{H}_{u} \mathbf{H}_{u}^{H} \boldsymbol{\beta}_{u}^{H}=\omega_{u}^{+}, \\
& \boldsymbol{\beta}_{u} \mathbf{H}_{u} \boldsymbol{\alpha}_{u}=\sqrt{\omega_{u}^{+}} .
\end{aligned}
$$

Two types of user-wise JTRD can be considered from a view point of procedure to determine JTRD weight vectors, $\boldsymbol{\alpha}_{u}$ and $\boldsymbol{\beta}_{u}$. It should be noted that type 1 and type 2 achieve the same received SNR since they can be described by the same transmission system model as illustrated in Fig. 1.

In type 1 , the $u$-th user determines $\boldsymbol{\beta}_{u}$ by solving the eigenvalue equation of Eq. (2) and subsequently, BS determines $\boldsymbol{\alpha}_{u}$. For BS to determine $\boldsymbol{\alpha}_{u}$, the knowledge of $\boldsymbol{\beta}_{u} \mathbf{H}_{u}$ is necessary. This is done as follows. The $u$-th user transmits the orthogonal pilot, which is precoded by $\boldsymbol{\beta}_{u}$, from its $N$ antennas. Then, BS receives it by $M$ antennas to acquire the knowledge of $\boldsymbol{\beta}_{u} \mathbf{H}_{u}$, from which $\boldsymbol{\alpha}_{u}$ is determined by using Eq. (1). After determining $\left\{\boldsymbol{\alpha}_{u} ; u=0 \sim U-1\right\}$, BS constructs the ZF precoder $\mathbf{W}_{\mathrm{ZF}}$. The $u$-th user requires only the knowledge of its own single-user MIMO channel $\mathbf{H}_{u}$ in order to construct the eigenvalue equation of Eq. (2). Furthermore, each user can equip an arbitrary number of antennas, but BS does not need to know each user's number of antennas.

On the other hand, in type 2 , the BS determines $\left\{\boldsymbol{\alpha}_{u} ; u=0 \sim U-1\right\}$ to construct $\mathbf{W}_{\mathrm{ZF}}$ by solving the eigenvalue equation of Eq. (3) and subsequently, the $u$-th user determines $\boldsymbol{\beta}_{u}$. For the $u$-th user to determine $\boldsymbol{\beta}_{u}$, the knowledge of $\mathbf{H}_{u} \boldsymbol{\alpha}_{u}$ is necessary. This is done as follows. BS transmits the orthogonal pilots, each precoded by $\boldsymbol{\alpha}_{u}, u=0 \sim U-1$, to $U$ users. Then, the $u$-th user receives the orthogonal pilot transmitted to it for acquiring the knowledge of $\mathbf{H}_{u} \boldsymbol{\alpha}_{u}$, from which $\boldsymbol{\beta}_{u}$ is determined by using Eq. (5). BS requires the knowledge of complete multi-user MIMO channel, but each user does not need to know the number of BS antennas.

\subsection{Design of ZF precoder/postcoder}

When using user-wise JTRD, the downlink composite multi-user MIMO channel is described by $\boldsymbol{\beta H} \boldsymbol{\alpha}$, where $\boldsymbol{\beta}=\operatorname{blockdiag}\left(\begin{array}{lllll}\boldsymbol{\beta}_{0} & \cdots & \boldsymbol{\beta}_{u} & \cdots & \left.\boldsymbol{\beta}_{U-1}\right) \text {. Since }\end{array}\right.$ $\boldsymbol{\beta H}=\sqrt{\boldsymbol{\omega}^{+}} \boldsymbol{\alpha}^{H}$ from Eq. (4), where $\boldsymbol{\omega}^{+}=\operatorname{diag}\left(\begin{array}{lllll}\omega_{0}^{+} & \cdots & \omega_{u}^{+} & \cdots & \omega_{U-1}^{+}\end{array}\right)$, a matrix inversion of $\boldsymbol{\alpha}^{H} \boldsymbol{\alpha}$ is introduced in order to remove the downlink multiuser interference. A concatenation of $\boldsymbol{\alpha}$ (in $\boldsymbol{\beta H} \boldsymbol{\alpha})$ and $\left(\boldsymbol{\alpha}^{H} \boldsymbol{\alpha}\right)^{-1}$ constitutes the ZF precoder $\mathbf{W}_{\mathrm{ZF}}=\boldsymbol{\alpha}\left(\boldsymbol{\alpha}^{H} \boldsymbol{\alpha}\right)^{-1}$. This is shown in Fig. 1.

Assuming TDD, the JTRD weight vectors associated with the $u$-th user for uplink transmission are given by $\boldsymbol{\alpha}_{u}^{T}$ and $\boldsymbol{\beta}_{u}^{T}$. Since the uplink composite multi-user MIMO channel is described by $(\boldsymbol{\beta H} \boldsymbol{\alpha})^{T}$, the ZF postcoder to remove the uplink multi-user interference is given by $\mathbf{W}_{\mathrm{ZF}}^{T}$.

\section{Analysis of received SNR}

The received SNR is analyzed for the given complete multi-user MIMO channel $\mathbf{H}$.

A simple but useful expression for the received SNR is derived for a 2-user case $(U=2)$. 


\subsection{Received SNR expression}

The transmit signal vector $\mathbf{x}=\left[\begin{array}{lllll}x_{0} & \cdots & x_{m} & \cdots & x_{M-1}\end{array}\right]^{T}$ with $x_{m}$ to be transmitted from the $m$-th BS antenna can be expressed as

$$
\mathbf{x}=\mathbf{W}_{\mathrm{ZF}} \sqrt{2 \boldsymbol{\pi}^{-1} \mathbf{P}} \mathbf{d},
$$

where $\mathbf{d}=\left[\begin{array}{lllll}d_{0} & \cdots & d_{u} & \cdots & d_{U-1}\end{array}\right]^{T}$ with $E\left[\left|d_{u}\right|^{2}\right]=1, \mathbf{P}=\operatorname{diag}\left(P_{0} \quad \cdots \quad P_{u}\right.$ $\left.\cdots \quad P_{U-1}\right)$, and $\pi^{-1}=\operatorname{diag}\left(\begin{array}{lllll}\pi_{0}^{-1} & \cdots & \pi_{u}^{-1} & \cdots & \pi_{U-1}^{-1}\end{array}\right)$ represent the transmit data symbol vector, the power allocation matrix, and the user-wise power normalization matrix, respectively.

Using $\boldsymbol{\beta H}=\sqrt{\boldsymbol{\omega}^{+}} \boldsymbol{\alpha}^{H}$ (obtained from Eq. (4)) and $\boldsymbol{\alpha}^{H} \mathbf{W}_{\mathbf{Z F}}=\mathbf{I}$ (identity matrix), the downlink received signal vector $\mathbf{r}=\left[\begin{array}{lllll}r_{0} & \cdots & r_{u} & \cdots & r_{U-1}\end{array}\right]^{T}$ can be expressed as

$$
\mathbf{r}=\sqrt{\boldsymbol{\omega}^{+}} \boldsymbol{\alpha}^{H} \mathbf{x}+\boldsymbol{\beta} \boldsymbol{\eta}=\sqrt{2 \boldsymbol{\omega}^{+} \boldsymbol{\pi}^{-1} \mathbf{P}} \mathbf{d}+\boldsymbol{\beta} \boldsymbol{\eta},
$$

where $\boldsymbol{\eta}=\left[\begin{array}{lllll}\boldsymbol{\eta}_{0}^{T} & \cdots & \boldsymbol{\eta}_{u}^{T} & \cdots & \boldsymbol{\eta}_{U-1}^{T}\end{array}\right]^{T}$ with $\boldsymbol{\eta}_{u}=\left[\begin{array}{llll}\eta_{0}^{(u)} & \cdots & \eta_{n}^{(u)} & \cdots\end{array}\right.$ $\left.\eta_{N-1}^{(u)}\right]^{T}$ is the noise vector and $\left\{\eta_{n}^{(u)} ; u=0 \sim U-1, n=0 \sim N-1\right\}$ are independent zero-mean complex Gaussian variables with variance $E\left[\left|\eta_{n}^{(u)}\right|^{2}\right]=2 N_{0} / T_{s}\left(N_{0}\right.$ and $T$ denote the noise power spectrum density and the data symbol length in time, respectively). Since $\left\|\boldsymbol{\beta}_{u}\right\|_{2}^{2}=1$, the noise variance remains unchanged after diversity combining.

We can show $\pi_{u}=\left\|\mathbf{w}_{u}\right\|_{2}^{2}$, where $\mathbf{w}_{u}$ is the $u$-th column vector of $\mathbf{W}_{\mathrm{ZF}}$. Hence, the $u$-th user's received SNR $\gamma_{u}$ is expressed as

$$
\gamma_{u}=\left(\omega_{u}^{+} /\left\|\mathbf{w}_{u}\right\|_{2}^{2}\right) \Gamma_{u}
$$

where $\Gamma_{u}=P_{u} \cdot T_{s} / N_{0}$ represents the transmit symbol energy-to-noise power spectrum density ratio $\left(E_{S} / N_{0}\right)$ associated with the $u$-th user.

On the other hand, the uplink received signal vector can be expressed by using Eq. (4) as

$$
\mathbf{r}=\left(\boldsymbol{\beta} \mathbf{H} \mathbf{W}_{\mathrm{ZF}}\right)^{T} \sqrt{2 \mathbf{P}} \mathbf{d}+\mathbf{W}_{\mathrm{ZF}}^{T} \boldsymbol{\xi}=\sqrt{2 \boldsymbol{\omega}^{+} \mathbf{P}} \mathbf{d}+\mathbf{W}_{\mathrm{ZF}}^{T} \xi,
$$

where $\xi=\left[\begin{array}{lllll}\xi_{0} & \cdots & \xi_{m} & \cdots & \xi_{M-1}\end{array}\right]^{T}$ is the noise vector with variance $E\left[\left|\xi_{m}\right|^{2}\right]$ $=2 N_{0} / T_{S}$. It can be understood from Eq. (11) that the noise variance associated with the $u$-th user is enhanced by a factor of $\left\|\mathbf{w}_{u}\right\|_{2}^{2}$. As a consequence, the uplink received SNR becomes the same as the downlink.

\subsection{Special case of $U=2$}

As understood from Eq. (10), the $u$-th user's received SNR is governed by the $u$-th column vector $\mathbf{w}_{u}$ of $\mathbf{W}_{\mathrm{ZF}}$. When $U=2$, we can show $\left\|\mathbf{w}_{u}\right\|_{2}^{2}=\left(1-\left|\sum_{m=0}^{M-1} \alpha_{m, 0} \alpha_{m, 1}^{*}\right|^{2}\right)^{-1}$ after some manipulations, where $\alpha_{m, u}$ is the $m$-th element (associated with the $m$-th BS antenna) of JTRD weight vector $\boldsymbol{\alpha}_{u}$. Consequently, we obtain the following simple but useful expression for the $u$-th user's received SNR.

$$
\gamma_{u}=\left(1-\left|\sum_{m=0}^{M-1} \alpha_{m, 0} \alpha_{m, 1}^{*}\right|^{2}\right) \omega_{u}^{+} \Gamma_{u}
$$


The received SNR is governed by the correlation between the JTRD weight vectors, $\boldsymbol{\alpha}_{1}$ and $\boldsymbol{\alpha}_{2}$. This fact can be exploited in designing the BS diversity scheme for a different user-wise JTRD in the future.

\section{Link capacity evaluation}

The sum link capacity $C_{\text {sum }}=\sum_{u=0}^{U-1} \log _{2}\left(1+\gamma_{u}\right)[\mathrm{bps} / \mathrm{Hz}]$ achievable by ZF-based multi-user MIMO coordinated with user-wise JTRD in an uncorrelated Rayleigh fading environment is evaluated by Monte-Carlo numerical computation. For comparison, the sum link capacity achievable by user-wise MRTCD is also evaluated. The user-wise MRTCD is based on the maximal-ratio diversity [2] and uses $\boldsymbol{\beta}_{u}$ with $\beta_{u, n}=\left(h_{u, m_{u}^{+}}^{(n)}\right)^{*} / \sqrt{\sum_{n=0}^{N-1}\left|h_{u, m_{u}^{+}}^{(n)}\right|^{2}}$, where $m_{u}^{+}=\underset{m=0 \sim M-1}{\arg \max } \sum_{n=0}^{N-1}\left|h_{u, m}^{(n)}\right|^{2}$. The cumulative distribution function (CDF) of the sum link capacity is plotted in Fig. 2 for $U=2 \sim 8$ when $M=8, N=2$, and $\Gamma_{u}=20 \mathrm{~dB}$ for all $u$. It can be seen from Fig. 2 that user-wise JTRD performs better than user-wise MRTCD and that as $U$ increases, the superiority of user-wise JTRD over user-wise MRTCD becomes more evident.

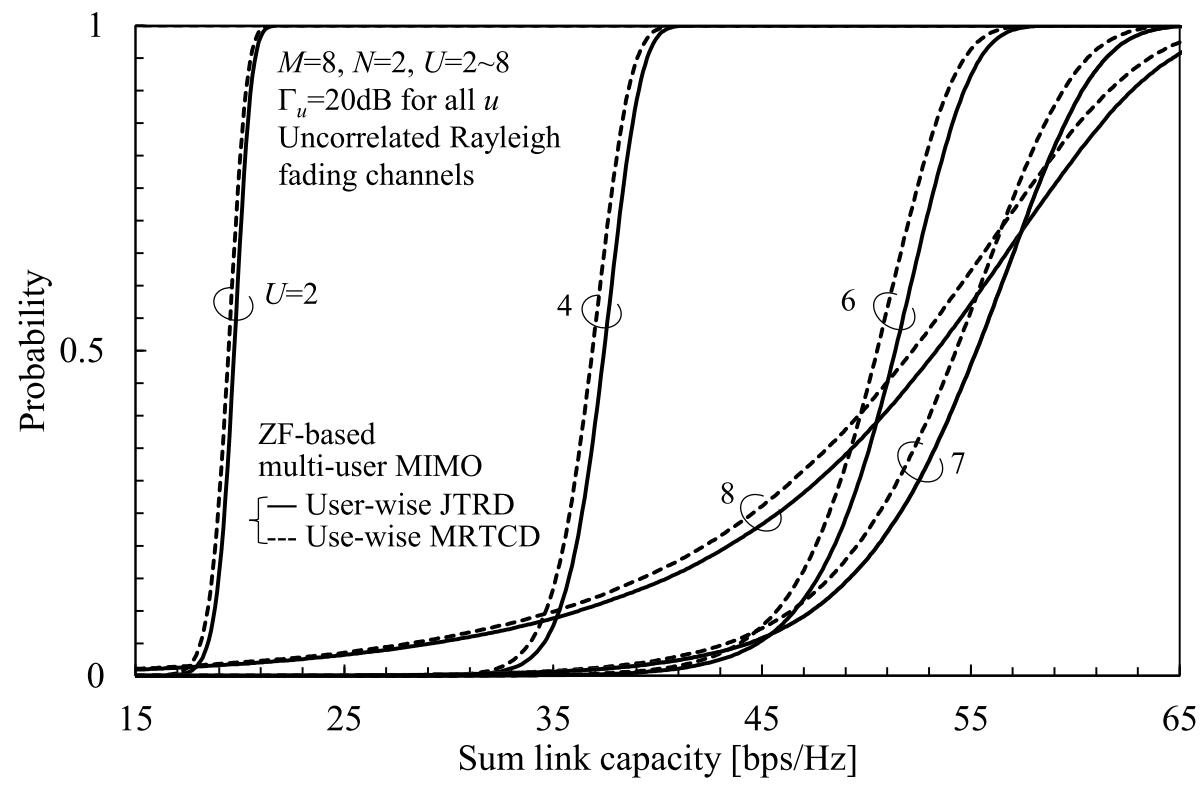

Fig. 2. Sum link capacity in an uncorrelated Rayleigh fading environment.

\section{Conclusions}

In this paper, user-wise JTRD was proposed for ZF-based multi-user MIMO using TDD. The sum link capacity in an uncorrelated Rayleigh fading environment was evaluated by Monte-Carlo numerical computation. It was shown that user-wise JTRD provides higher link capacity than user-wise MRTCD.

In this paper, uncorrelated fading was assumed. When BS equips a large number of antennas, e.g. array antenna, BS antennas experience correlated fading. Analysis of user-wise JTRD in such a case is left as an interesting study. In this paper, transmit 
power control (TPC) was not considered. If TPC is designed to keep the received SNR (or the link capacity) at a prescribed target value, the transmit $E_{S} / N_{0}$ varies due to fading. An evaluation of transmit $E_{S} / N_{0}$ distribution when TPC is adopted is also left as a future study.

\section{Acknowledgments}

A part of this work was conducted under " $R \& D$ for further advancement of the 5th generation mobile communication system" (JPJ000254) commissioned by the Ministry of Internal Affairs and Communications in Japan. 